\title{
openheart Comparison of left ventricular rotational mechanics between term and extremely premature infants over the first week of age
}

\author{
Aisling Smith, ${ }^{1}$ Neidin Bussmann, ${ }^{1}$ Phillip Levy, ${ }^{2}$ Orla Franklin, ${ }^{3}$ Naomi McCallion, ${ }^{1}$ \\ Afif EL-Khuffash (1D ${ }^{1,4}$
}

To cite: Smith A, Bussmann N, Levy $\mathrm{P}$, et al. Comparison of left ventricular rotational mechanics between term and extremely premature infants over the first week of age. Open Heart 2021;8:e001458. doi:10.1136/ openhrt-2020-001458

Received 22 September 2020 Revised 19 December 2020 Accepted 15 January 2021
Check for updates

(c) Author(s) (or their employer(s)) 2021. Re-use permitted under CC BY-NC. No commercial re-use. See rights and permissions. Published by BMJ.

${ }^{1}$ Neonatology, Rotunda Hospital, Dublin, Ireland

${ }^{2}$ Department of Pediatrics, Boston Children's Hospital, Boston, Massachusetts, USA ${ }^{3}$ Paediatric Cardiology, Our Lady's Children's Hospital Crumlin, Dublin, Ireland

${ }^{4}$ Department of Pediatrics, Royal College of Surgeons in Ireland, Dublin, Ireland

Correspondence to Professor Afif EL-Khuffash; afif_ faisal@hotmail.com

\section{ABSTRACT}

Objective Left ventricle (LV) rotational mechanics is an emerging tool to characterise LV function, but warrants further evaluation in neonates. The aim of this study was to compare LV rotational mechanics between term and extremely preterm babies over the first week of age.

Methods In this prospective study, we serially assessed LV rotational parameters in 50 term infants and compared them with a historical dataset of 50 preterm infants born $<29$ weeks gestation. LV basal and apical rotation, LV twist, LV twist/untwist rate and torsion were derived using two-dimensional speckle tracking echocardiography at three time points over the first week of age.

Results There was no change in LV twist, LV torsion, basal rotation or apical rotation in term infants over the study period (all $p>0.05$ ). LV twist and torsion were higher in preterm infants, and increased over time. In preterm infants, basal rotation evolved from anticlockwise to clockwise rotation. Apical rotation remained anticlockwise in both groups (all $p>0.05$ ). LV twist rate (LVTR) and untwist rate was higher in preterm infants and increased over the three time points (all $p>0.05$ ). There was a strong positive correlation between LV torsion and LV untwist rate (LVUTR) in the entire cohort during the third scan.

Conclusion Term infants exhibit minimal LV twist which remains unchanged over the first week of age. This is in contrast to premature infants who demonstrate increasing indices of twist, torsion, LVTR and LVUTR over the first week, likely as a compensatory mechanism for reduced LV compliance.

\section{INTRODUCTION}

Left ventricular ( $\mathrm{LV})$ performance is a major prognostic determinant of cardiopulmonary pathologies in neonates. ${ }^{1}$ The LV myocardium is comprised circumferential fibres in the mid-wall layer and longitudinal fibres in the endocardial and epicardial layer that collectively contribute to its complex contraction patterns. ${ }^{2}$ The helical configuration changes continuously from a right-handed orientation in subendocardium to a left-handed orientation in subepicardium granting the LV

\section{Key questions}

What is already known about this subject?

- Premature babies exhibit higher indices of left ventricle (LV) twist and untwist in the early newborn period than infants born at term.

What does this study add?

- This study provides reference patterns of LV rotational mechanics in healthy term infants over the few days of age and demonstrates a lack on LV twist, LV torsion, basal and apical rotation in this population.

How might this impact on clinical practice?

- This study highlights inherent differences in myocardial function between preterm and term infants shortly after birth and provides an in depth understanding of the trajectory of LV performance in neonates. Further studies are now needed to investigate the impact LV rotational mechanics has on shortterm and long-term outcomes in different neonatal disease states.

unique rotational properties. ${ }^{3}$ The base of the heart rotates in a clockwise motion while the apex of the heart rotates in an anticlockwise motion. The net effects of these two opposing forces is myocardial twist, a 'wringing' motion of the $\mathrm{LV}$, is thought to support both systolic and diastolic performance. ${ }^{4}$ In systole, LV twist will store potential energy and aid ejection of blood from the ventricle. In diastole the recoil of the $\mathrm{LV}$ and release of potential energy as it returns to its baseline untwisted shape creates a negative pressure gradient and suction-like action supporting early diastolic filling. ${ }^{5}$ Myocardial twist may act as a compensatory mechanism for reduced LV elasticity by minimising transmural stress of the LV muscle, supporting impaired systolic or diastolic function and helping to maintain adequate cardiac output. ${ }^{6}$ 
There are distinct developmental differences in LV myofibre structure and function between term and extremely premature infants that can be characterised by rotational properties over the first days of age, but warrants further comparative study. The myocardium of premature neonates is less compliant than that of term neonates, likely due to the relatively high content of collagen and high ratio of type I to type III collagen. ${ }^{7}$ The unique cardiac phenotype of premature infants is known to persist into childhood as a recent meta-analysis demonstrated that preterm-born individuals have persistently smaller ventricular dimensions, ongoing systolic and diastolic dysfunction that worsens with age, and an accelerated rate of LV hypertrophy from childhood to adulthood. ${ }^{8}$ Our group has explored the natural progression of rotational mechanics in preterm infants immediately after birth, ${ }^{1}$ but the interpretation of the results has been lacking without serial comparisons to term infants.

The recent development of two-dimensional speckle tracking echocardiography (2DSTE) measured rotational parameters provide clinicians with an efficient and reliable non-invasive modality to characterise LV performance in neonates. ${ }^{4}$ As such, we hypothesised that the LV twist and untwist mechanisms derived by 2DSTE would differ between healthy infants born at term and infants born at extremely premature gestations. The aim of this study was to compare LV rotational mechanics between term and preterm babies over the first week of age.

\section{METHODS}

\section{Design and study population}

In this prospective cohort study we enrolled 50 healthy term infants born at the Rotunda Hospital, Dublin Ireland between September 2018 and August 2019. We compared the term group with a historical premature infant population that was prospectively enrolled and has previously been described by our group. ${ }^{1}$ Healthy term infants were defined as appropriately grown infants $(>10$ th centile and $<90$ th centile for birth weight) delivered between $37^{+0}$ and $41^{+0}$ weeks gestation. Specific exclusion criteria for the term cohort consisted of any infant with antenatal or postnatal evidence of congenital or chromosomal anomalies, maternal diabetes mellitus of any type, preeclampsia, maternal hypertension, clinical or suspected chorioamnionitis, or evidence of absent or reversed end diastolic flow in the umbilical arteries anytime during the pregnancy. Infants with perinatal acidosis (umbilical cord $\mathrm{pH}<7.10$ ), neuromuscular disorders, abnormal cardiac rhythms and infants admitted to the neonatal intensive care unit (NICU) for any reason were also excluded from the healthy term population. We included a larger proportion of term infants born via caesarean section to optimise obtaining third echocardiogram during their hospital stay at a time that resembles the timing of the third scan of the premature infant population. Term infants born via caesarean remain in our hospital for up to 5 days following birth.
The historical preterm population consisted of 50 infants born $<29$ weeks gestation admitted to the NICU between January and December 2013. ${ }^{1}$ Premature infants were excluded if they were small for gestational age $(<10$ th centile), received any inotropes or inhaled nitric oxide, died within the first 7 days of age or had any evidence of congenital heart disease (other than a patent ductus arteriosus, PDA) or congenital/chromosomal anomalies. During that time, our unit adopted a conservative approach to PDA treatment in premature infants. Medical prophylactic and early PDA treatment was not practised. Basic clinical information and delivery details were collected for both groups.

\section{Patient and public involvement statement}

Written informed consent was obtained from parents prior to enrolment.

\section{Echocardiography assessments}

We performed serial echocardiograms at three time points after delivery: Echocardiogram 1 (median of 14 hours (9-22)), Echocardiogram 2 (44 hours (40-47)) and Echocardiogram 3 (117 hours (72-148)) using the General Electric Echocardiography system (Vivid I, Vivid S6 and Vivid E95; GE Healthcare, Milwaukee, Wisconsin, USA). All studies were conducted using a standardised imaging protocol in accordance with published guidelines specific to the neonatal population. ${ }^{910} \mathrm{~A}$ full structural assessment was carried out during the first echocardiogram to rule out the presence of congenital heart disease. The images were stored on an archiving system in raw format (DICOM) for later offline analysis. All images were analysed using the GE EchoPac system (V.112).

Tissue Doppler imaging (TDI) of the left (LV) and right (RV) ventricular systolic $\left(\mathrm{s}^{\prime}\right)$ and diastolic $\left(\mathrm{e}^{\prime}\right.$ and $\left.\mathrm{a}^{\prime}\right)$ velocities $(\mathrm{cm} / \mathrm{s})$ from the base of the $\mathrm{LV}$ free wall and the RV free wall, respectively in the apical four chamber view while maintaining and angle of insonation $<20^{\circ}$ at all times were collected in accordance with published methodology. ${ }^{11}$ We recorded systolic and diastolic times (ms) from the base of the LV free wall using TDI. PDA characteristics included diameter $(\mathrm{mm})$, maximum systolic velocity $(\mathrm{m} / \mathrm{s})$, left atrial to aortic root ratio (LA:Ao), and the presence of bidirectional shunting were measured in addition to mitral valve $\mathrm{E}$ wave to $\mathrm{A}$ wave ratio and $\mathrm{LV}$ ejection fraction. $^{12}$

We used pulmonary artery acceleration time (PAAT) as a surrogate measurement for assessment of pulmonary vascular resistance (PVR) and right ventricular afterload. PAAT adjusted for right ventricular ejection time (RVET) has been validated against right heart catheterisation as a reliable estimate of pulmonary haemodynamics in children and neonates. ${ }^{13}$ Our group has demonstrated the reliability and maturational changes of this measurement in the preterm and term neonatal population. ${ }^{14-16}$ PAAT and RVET were obtained by placing a pulsed wave Doppler sample at the level of the pulmonary artery annulus in the long axis parasternal view. To account 
for heart rate variability between the two groups, PAAT was adjusted for RVET using the PAAT/RVET ratio (and termed PAATi).

LV rotational mechanics including apical and basal rotation, $\mathrm{LV}$ twist, $\mathrm{LV}$ torsion, $\mathrm{LV}$ twist rate (LVTR) and LV untwist rate (LVUTR) were obtained from the parasternal short axis view of the LV at the levels of the apex and mitral valve. Rotation was characterised as the circumferential clockwise (negative rotation) or anticlockwise (positive rotation) movement of the base and apex which occurs during systole and is measured in degrees. Twist was defined as the net difference between apical and basal rotation and is measured in degrees. $\mathrm{LV}$ torsion was defined as LV twist indexed to LV length; facilitating the comparison of $\mathrm{LV}$ rotational mechanics across varying $L V$ sizes. LVTR is the speed at which twist occurs during systolic while LVUTR describes the speed at which untwisting occurs during early diastole. LV untwist is usually depicted as a negative movement to indicate a return to baseline; we elected to present absolute LVUTR values to simplify graphical presentation. Detailed methodology of image acquisition and measurement of the various parameters including validity and reproducibility are published by our group elsewhere. ${ }^{14}$ We and others have previously demonstrated excellent reproducibility with rotational mechanics in term and preterm infants. ${ }^{1}{ }^{17-19}$

\section{Statistical analysis}

Continuous data were assessed for normality using the Shapiro-Wilk test and a histogram representation and summarised as means ( $\mathrm{SD}$ ) or medians (IQR) as appropriate. The two groups were compared using the Student's t-test of the Mann-Whitney U test as appropriate. Categorical variables were presented as absolute count (per cent) and compared using the $\chi^{2}$ test or the Fisher exact test as appropriate. Correlations were tested using Pearson's correlation coefficient. Linear regression was used to assess the relationship between heart rate and torsion on the third scan while controlling for gestation. Two-way analysis of variance (ANOVA) was used to compare change in rotational mechanics measurements over time in the two groups. A p value $<0.05$ was considered significant. SPSS (IBM, V.26) was used to conduct the statistical analysis.

\section{RESULTS}

\section{Baseline characteristics}

The demographic and clinical characteristics between the term infants and preterm infants are presented in table 1 . There was no difference in sex or the rate of caesarean section between the two groups. The 5 min Apgar score was slightly lower and cord $\mathrm{pH}$ slightly higher in the preterm group.

\section{Conventional echocardiography measurements}

Term infants had a lower heart rate on day 1, longer diastolic time and a lower SD ratio. PAAT and RVET

\begin{tabular}{|c|c|c|c|}
\hline & $\begin{array}{l}\text { Term group } \\
n=50\end{array}$ & $\begin{array}{l}\text { Preterm group } \\
\mathrm{n}=50\end{array}$ & $P$ value \\
\hline $\begin{array}{l}\text { Gestation } \\
\text { (weeks) }\end{array}$ & 39.5 (38.9-40.6) & $27.0(25.8-28.0)$ & $<0.01$ \\
\hline $\begin{array}{l}\text { Birth weight } \\
\text { (g) }\end{array}$ & $3600(3258-3938)$ & $885(758-1143)$ & $<0.01$ \\
\hline Male & $19(38)$ & $28(56)$ & 0.11 \\
\hline $\begin{array}{l}\text { Caesarean } \\
\text { section }\end{array}$ & $31(62)$ & $38(76)$ & 0.19 \\
\hline $\begin{array}{l}5 \text { min Apgar } \\
\text { score }\end{array}$ & $10(9-10)$ & $9(8-9)$ & $<0.01$ \\
\hline Cord pH & $7.31(7.28-7.34)$ & 7.35 (7.32-7.37) & $<0.01$ \\
\hline
\end{tabular}

Values are presented as medians (IQR) or count (\%).

were higher in the term infants; however, there was no difference in PAATi between the two groups on day 1 (table 2). Term infants had higher LV systolic and diastolic tissue-Doppler velocities, and higher LV ea' ratio when compared with preterm infants (table 2). Similarly, term infants possessed higher RV systolic, early and late diastolic tissue Doppler velocities when compared with preterm infants (table 2). There was a higher rate of PDAs in the preterm infants on day 1 and a larger PDA diameter; there was no difference in the maximum systolic velocity across the PDA or the rate of bidirectional shunting between the two groups (table 2). By the third echocardiogram $4(8 \%)$ infants in the term group still had a PDA (vs $34(68 \%))$ in the preterm group, $(\mathrm{p}<0.01)$, with none having a bidirectional shunt (vs 5/34 (15\%)) in the preterm group, $(p=1.0)$. LA:Ao was higher in the preterm group by the third echocardiogram $(1.7 \pm 0.4$ vs $1.4 \pm 0.2, \mathrm{p}=0.01$ ).

\section{Comparison of rotational mechanics}

The changes in the rotational mechanics in the two groups over the study period are illustrated in figure 1 . There was no change in LV twist or torsion in the term group over the three time points. In the preterm group, LV twist and torsion were higher than the term group over the three time points and increased over time (figure 1A). There was no change in basal rotation in the term group over the study period. In the preterm group, basal rotation was positive (anticlockwise) during Echocardiogram 1, changing to negative (clockwise) by Echocardiogram 3 with a significant change over time. Apical rotation remained positive (anticlockwise) and similar between the two groups with no change over the study period (figure 1B). In the preterm group LVTR increased over time and became higher than the term group by the third echocardiogram; LVUTR was increased in the preterm group over time and was higher than the term group during the second and third scan. There was no change in LVTR and untwist rate in the term group over the study period (figure 1C). There was a strong positive correlation between LV torsion 
Table 2 Conventional echocardiography measurements on day 1

\begin{tabular}{|c|c|c|c|}
\hline & $\begin{array}{l}\text { Term group } \\
n=50\end{array}$ & $\begin{array}{l}\text { Preterm group } \\
n=50\end{array}$ & $\begin{array}{l}P \\
\text { value }\end{array}$ \\
\hline Heart rate & $128 \pm 15$ & $156 \pm 12$ & $<0.01$ \\
\hline Systolic time (ms) & $190 \pm 20$ & $139 \pm 15$ & $<0.01$ \\
\hline Diastolic time (ms) & $193 \pm 45$ & $122 \pm 17$ & $<0.01$ \\
\hline SD ratio & $1.0 \pm 0.2$ & $1.2 \pm 0.2$ & $<0.01$ \\
\hline $\begin{array}{l}\text { Pulmonary artery } \\
\text { acceleration time (ms) }\end{array}$ & $62 \pm 15$ & $41 \pm 9$ & $<0.01$ \\
\hline $\begin{array}{l}\text { Right ventricular } \\
\text { ejection time (ms) }\end{array}$ & $211 \pm 23$ & $151 \pm 22$ & $<0.01$ \\
\hline PAATi & $0.29 \pm 0.06$ & $0.28 \pm 0.06$ & 0.15 \\
\hline $\begin{array}{l}\text { Left ventricular length } \\
(\mathrm{mm})\end{array}$ & $31 \pm 2$ & $18 \pm 2$ & $<0.01$ \\
\hline $\mathrm{LV} \mathrm{s}^{\prime}(\mathrm{cm} / \mathrm{s})$ & $4.9 \pm 1.1$ & $2.8 \pm 0.8$ & $<0.01$ \\
\hline $\mathrm{LV}^{\prime}(\mathrm{cm} / \mathrm{s})$ & $6.5 \pm 1.5$ & $3.7 \pm 1.4$ & $<0.01$ \\
\hline $\mathrm{LV} \mathrm{a}^{\prime}(\mathrm{cm} / \mathrm{s})$ & $5.7 \pm 1.7$ & $4.5 \pm 1.8$ & $<0.01$ \\
\hline LV ea' ratio & $1.2 \pm 0.3$ & $0.9 \pm 0.3$ & $<0.01$ \\
\hline Ejection fraction (\%) & $60 \pm 5$ & $60 \pm 6$ & 0.64 \\
\hline Mitral valve E:A & $1.1 \pm 0.3$ & $0.7 \pm 0.1$ & $<0.01$ \\
\hline $\mathrm{RV} \mathrm{s}^{\prime}(\mathrm{cm} / \mathrm{s})$ & $6.3 \pm 1.5$ & $3.8 \pm 0.9$ & $<0.01$ \\
\hline $\mathrm{RV} \mathrm{e}^{\prime}(\mathrm{cm} / \mathrm{s})$ & $8.7 \pm 1.6$ & $4.3 \pm 1.4$ & $<0.01$ \\
\hline $\mathrm{RV} \mathrm{a}^{\prime}(\mathrm{cm} / \mathrm{s})$ & $8.9 \pm 2.2$ & $7.4 \pm 1.9$ & $<0.01$ \\
\hline PDA & $26(52)$ & $47(94)$ & $<0.01$ \\
\hline PDA diameter (mm) & $1.9(1.6-2.5)$ & $2.4(2.1-2.9)$ & $<0.01$ \\
\hline $\begin{array}{l}\text { PDA maximum systolic } \\
\text { velocity }(\mathrm{m} / \mathrm{s})\end{array}$ & $1.1(-1.1-2.0)$ & $1.1(0.8-1.6)$ & 0.64 \\
\hline LA:A0 & $1.4 \pm 0.2$ & $1.4 \pm 0.2$ & 0.93 \\
\hline Bidirectional shunting & 10/26 (39) & $19 / 47(40)$ & 1.00 \\
\hline
\end{tabular}

Values are presented as means (SD), medians (IQR) or count (\%).

and LVUTR in the term and preterm infants during the third scan (figure 2). Within each group, there was no correlation between heart rate or any of the rotational measurements. On linear regression, there was no relationship between torsion on the third scan and heart rate in the entire cohort (standardised $\beta-0.1, p=0.48$ ) while controlling for gestation (the main predictor: standardised $\beta-0.7, p<0.01)$. In the preterm infant group, there was no correlation between PDA diameter or LA:Ao and any of the rotational measurements (all $\mathrm{p}>0.05$ ).

\section{Summary of findings}

In this single-centre prospective observational study of healthy term infants, normative patterns of rotational mechanics were assessed and compared with a historical cohort of extremely preterm infants using 2DSTE over the first few days of age. The study revealed no change in basal rotation, apical rotation, LV twist, LV torsion, LVTR or LVUTR in the term group after delivery. In contrast, the higher indices of twist, twist/untwist rate and torsion
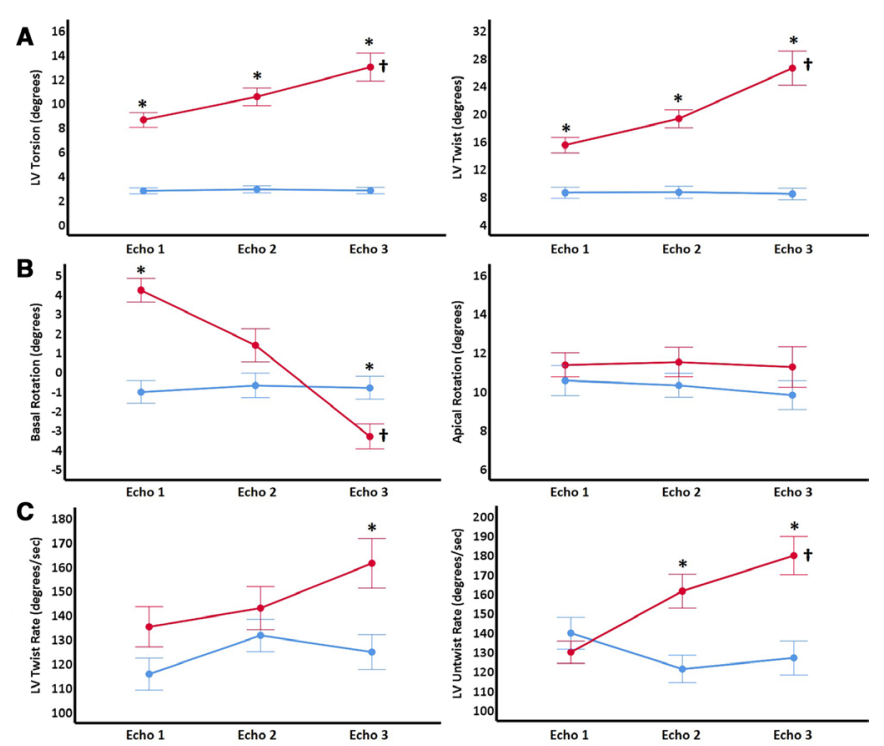

Figure 1 Comparison of rotation mechanics between term (blue) and preterm (red) infants. Values are presented as means (diamonds) and one SE (whiskers). ${ }^{*} P$ value $<0.05$ at the time point. †Significant change overtime in that group. LV, left ventricle.

observed in the preterm group were driven by a change in basal rotation from an anticlockwise to clockwise motion over the first week of age as previously described. Notably, term infants had superior LV and RV function compared with the premature cohort as illustrated by higher systolic and diastolic tissue Doppler velocities. In particular, term infants demonstrated much more favourable diastolic performance characterised by a higher LV ea' ratio on tissue Doppler, a higher mitral valve E:A wave ratio on pulsed wave Doppler, and a lower systolic to diastolic ratio. In addition, similar PAATi and PDA data indicate that the relative degree of PVR experienced by both the premature and term cohorts was equivalent. The inherent differences in LV performance, as measured by rotational mechanics between preterm and term infants shortly after birth provides an in depth understanding of the trajectory of LV performance during the transitional period and lays the foundation to design therapeutic models that will use of these measures to assess

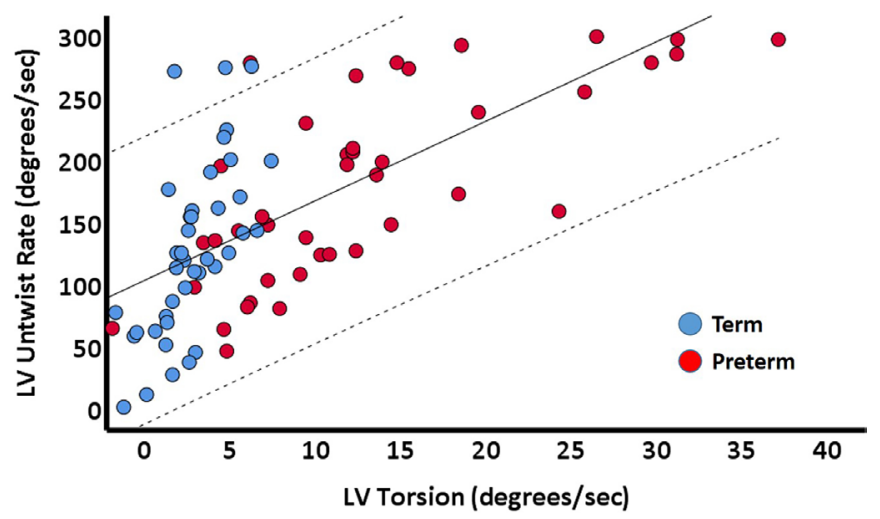

Figure 2 Correlation between LV torsion and LV untwist rate over in the entire cohort over on day 3 . LV, left ventricle. 
the efficacy of management strategies within different neonatal disease states.

The study highlights the relative lack of LV twist and torsion exhibited by healthy term infants after delivery. The term neonatal myocardium is conditioned to handle changes in loading conditions more efficiently than the preterm myocardium and we observed preservation of the systolic and diastolic function immediately after birth. The higher diastolic function indices seen in term infants further suggest more ideal ventricular relaxation properties driven by more mature compliant ventricles. In contrast, decreased biventricular function and associated augmentation of rotational mechanics evident in the premature population supports the notion of LV twist acting as a compensatory mechanism for compromised longitudinal systolic and (in particular) diastolic function. Premature infants exhibit a stiffer, less compliant myocardium than their term counterparts and associated diastolic dysfunction pervades within this population. ${ }^{8}$ It is known that LV twist in systole is an important element influencing LV diastolic filling through LV energy recoil. ${ }^{20}$ Therefore, the lack of twist and untwist rates visualised in our term group are likely supporting the notion that rotational mechanics act a compensatory mechanism when LV diastolic function is compromised. The higher indices of twist and torsion seen in the premature population are due to the change in basal rotation from an anticlockwise to clockwise motion. This was also reported in preterm infants by Castaldi $e t a l .^{21}$ This concept is further reinforced with the strong positive correlation between $\mathrm{LV}$ torsion and untwist rate in the entire cohort during the third scan in this study. The adaptive response of LV twist in our premature cohort most likely evolved due to diminished longitudinal function and not consequent to any interplay with pulmonary haemodynamics, since the relative indices of PVR were equivalent between preterm and term babies.

The physiological basis for LV twist lies within the orientation of the myocardial fibres and the balance which exists between the positive torsional forces of the left handed, subepicardial fibres and the negative torsional forces of the right handed, subendocardial fibres. ${ }^{1}$ Dominant, positive subepicardial deformation is typically opposed by negative subendocardial deformation; however, high afterload environments are known to attenuate subendocardial motion. ${ }^{22}$ In the context of premature birth, when the low resistance placenta is removed at delivery, the $\mathrm{LV}$ is suddenly exposed to both a rapid increase in afterload and a rapid decrease in preload, especially if delayed cord clamping is not performed or contraindicated. The increase in LV rotational parameters observed in our findings over the first week of age may represent the premature LV adapting to its new postnatal environment of high afterload. High LV afterload may mitigate subendocardial fibre contraction leading to a relative increase in unopposed epicardial, positive deformation and an overall net effect of increasingly positive twist. ${ }^{23}$ The lack of association between PDA diameter or LA:Ao ratio and the rotational measurements in the premature infant group further suggests that increased LV preload that may be experienced in the setting of a persistent ductus does not influence twist or torsion in this population.

A recent study of 117 infants born prematurely at $<29$ weeks gestation with longitudinal follow-up documented decreasing torsion from 32 weeks corrected age to 1 year corrected age in all preterm infants $(\mathrm{p}<0.001) .{ }^{17}$ This decline in torsion was due to alterations in the direction and magnitude of apical rotation with age. Although twist was required in early premature life, these findings suggest it is not as critical as the infant ages and that their myocardium is capable of recovery. Adequate myocardial performance in the absence of twist over time can be achieved. ${ }^{17}$ In such a circumstance the lack of twist demonstrated in the developing ex-premature heart with age is akin to the state of a healthy infant born at term.

\section{Clinical importance of rotational mechanics in neonates}

The benefit of LV rotation measurements obtained via 2DSTE lies in it's ability to non-invasively assess myocardial performance at the bedside, in real time, and provide insights into diastolic function. ${ }^{24} \mathrm{LV}$ rotational mechanics is a reliable and sensitive method for diagnosing neonatal diseased states, monitoring the progression of illness, evaluating therapeutic response and providing important prognostic information. Several recent studies performed in neonates have highlighted the growing recognition that LV twist represents a natural compensatory response to decreased myocardial function in pathological conditions. ${ }^{1}{ }^{17-19}$ Similarly mechanistic insights have been learnt from adult studies as Zhang et al reported significant increases in peak twist and basal rotation in people over 65 years old, likely as an adaptive response to age related myocardial decline. ${ }^{25} 26$ Bertini et al explored reported that peak LV twist was reduced in patients with acute myocardial infarction and in those with heart failure. ${ }^{27} \mathrm{~A}$ strong correlation $(\mathrm{r}=0.87$, $\mathrm{p}<0.001$ ) was documented between peak LV twist and LV ejection fraction in the overall study population stressing the importance of $\mathrm{LV}$ twist as an integral mechanism supporting normal cardiac output.

\section{LIMITATIONS}

The gold standard approach for LV rotational mechanics is three-dimensional STE or cardiac MRI. However, the use of 2DSTE, a relatively angle-independent and widely available tool at the bedside, has been validated and demonstrated to be both feasible and reproducible in term and preterm neonates. ${ }^{14}$ 17-19 The comparison of relatively healthy term infants to a historical cohort of uncomplicated preterm infants should be taken into account when applying the data. Future work is needed to build on comparison efforts in different neonatal disease states. 


\section{CONCLUSIONS}

In conclusion, our observational study serially assessing $\mathrm{LV}$ rotational mechanics in 50 infants born at term compared with a historical cohort of 50 infants born $<29$ weeks gestation has demonstrated that term infants do not exhibit LV twist and maintain static LV twist and untwist rates over the first week of age. In contrast premature infants demonstrate increasing indices of twist, torsion, LVTR and LVUTR over this time frame. With established reference patterns of rotational mechanics in term and preterm infants over the first week of age, future work can further elucidate physiological mechanisms of myocardial function, evaluate the degree of diastolic dysfunction in premature infants, assess disease severity, monitor treatment response, predict short term outcomes and identify those at risk for myocardial impairment past the neonatal period into childhood and beyond.

\section{Twitter Afif EL-Khuffash @afif_elkhuffash}

Contributors AS: planning, conducting; patient enrolment, data collection and reporting; article writing, submission. NB: conducting; patient enrolment, data collection. NM: reporting; article writing, data check, statistical check. PL: article writing, data check. OF: article writing, statistical analysis. AEK: planning; principal investigator, reporting; article writing.

Funding This project was funded by The National Children's Hospital Foundation/ Health Research Board Research Grant (Ref: NCHF-2017-005). The National Children's Research Centre funded PhD fees associated with this work.

Competing interests None declared.

Patient consent for publication Not required.

Ethics approval The study was approved by the medical ethics committee of the hospital and all investigators adhered to the principles of the Declaration of Helsinki.

\section{Provenance and peer review Not commissioned; externally peer reviewed.}

Data availability statement No data are available due to GDPR restrictions.

Open access This is an open access article distributed in accordance with the Creative Commons Attribution Non Commercial (CC BY-NC 4.0) license, which permits others to distribute, remix, adapt, build upon this work non-commercially, and license their derivative works on different terms, provided the original work is properly cited, appropriate credit is given, any changes made indicated, and the use is non-commercial. See: http://creativecommons.org/licenses/by-nc/4.0/.

ORCID iD

Afif EL-Khuffash http://orcid.org/0000-0002-2933-822X

\section{REFERENCES}

1 James A, Corcoran JD, Mertens L, et al. Left ventricular rotational mechanics in preterm infants less than 29 weeks' gestation over the first week after birth. J Am Soc Echocardiogr 2015;28:808-17.

2 Greenbaum RA, Ho SY, Gibson DG, et al. Left ventricular fibre architecture in man. Br Heart J 1981;45:248-63.

3 Ingels NB. Myocardial fiber architecture and left ventricular function. Technol Health Care 1997;5:45-52.

4 El-Khuffash A, Schubert U, Levy PT, et al. Deformation imaging and rotational mechanics in neonates: a guide to image acquisition, measurement, interpretation, and reference values. Pediatr Res 2018;84:30-45.

5 Nakatani S. Left ventricular rotation and twist: why should we learn? J Cardiovasc Ultrasound 2011;19:1-6.

6 Stöhr EJ, Shave RE, Baggish AL, et al. Left ventricular twist mechanics in the context of normal physiology and cardiovascular disease: a review of studies using speckle tracking echocardiography. Am J Physiol Heart Circ Physiol 2016;311:H633-44.

7 Marijianowski MM, van der Loos CM, Mohrschladt MF, et al. The neonatal heart has a relatively high content of total collagen and type I collagen, a condition that may explain the less compliant state. $J$ Am Coll Cardiol 1994;23:1204-8.

8 Telles F, McNamara N, Nanayakkara S, et al. Changes in the preterm heart from birth to young adulthood: a meta-analysis. Pediatrics. In Press 2020;146:1-3. doi:10.1542/peds.2020-0146

9 de Boode WP, Singh Y, Gupta S, et al. Recommendations for neonatologist performed echocardiography in Europe: consensus statement endorsed by European Society for paediatric research (ESPR) and European Society for neonatology (ESN). Pediatr Res 2016;80:465-71.

10 Groves AM, Singh Y, Dempsey E, et al. Introduction to neonatologistperformed echocardiography. Pediatr Res 2018;84:1-12.

11 Nestaas E, Schubert U, de Boode WP, et al. Tissue Doppler velocity imaging and event timings in neonates: a guide to image acquisition, measurement, interpretation, and reference values. Pediatr Res 2018;84:18-29.

12 van Laere D, van Overmeire B, Gupta S, et al. Application of NPE in the assessment of a patent ductus arteriosus. Pediatr Res 2018;84:46-56.

13 Levy PT, Patel MD, Groh G, et al. Pulmonary artery acceleration time provides a reliable estimate of invasive pulmonary hemodynamics in children. J Am Soc Echocardiogr 2016;29:1056-65.

14 Patel MD, Breatnach CR, James AT, et al. Echocardiographic assessment of right ventricular afterload in preterm infants: maturational patterns of pulmonary artery acceleration time over the first year of age and implications for pulmonary hypertension. J Am Soc Echocardiogr 2019;32:884-94.

15 Jain A, Mohamed A, El-Khuffash A, et al. A comprehensive echocardiographic protocol for assessing neonatal right ventricular dimensions and function in the transitional period: normative data and Z scores. J Am Soc Echocardiogr 2014;27:1293-304.

16 Jain A, Mohamed A, Kavanagh B, et al. Cardiopulmonary adaptation during first day of life in human neonates. J Pediatr 2018;200:50-7.

17 Lehmann GC, Levy PT, Patel MD, et al. Maturational patterns of left ventricular rotational mechanics in pre-term infants through 1 year of age. Cardiol Young 2020;30:1-9.

18 Breatnach CR, Forman E, Foran A, et al. Left ventricular rotational mechanics in infants with hypoxic ischemic encephalopathy and preterm infants at 36 weeks postmenstrual age: A comparison with healthy term controls. Echocardiography 2017;34:232-9.

19 Maskatia SA, Lee W, Altman CA, et al. Left ventricular rotational mechanics in early infancy: normal reference ranges and reproducibility of peak values and time to peak values. Early Hum Dev 2017;104:39-44.

20 Boissière $\mathrm{J}$, Maufrais $\mathrm{C}$, Baquet $\mathrm{G}$, et al. Specific left ventricular twist-untwist mechanics during exercise in children. J Am Soc Echocardiogr 2013;26:1298-305.

21 Castaldi B, Bordin G, Favero V, et al. Early modifications of cardiac function in preterm neonates using speckle tracking echocardiography. Echocardiography 2018;35:849-54.

22 MacGowan GA, Burkhoff D, Rogers WJ, et al. Effects of afterload on regional left ventricular torsion. Cardiovasc Res 1996;31:917-25.

23 A'roch R, Gustafsson U, Poelaert J, et al. Left ventricular twist is load-dependent as shown in a large animal model with controlled cardiac load. Cardiovasc Ultrasound 2012;10:26.

24 Mawad W, Friedberg MK. The continuing challenge of evaluating diastolic function by echocardiography in children: developing concepts and newer modalities. Curr Opin Cardiol 2017;32:93-100.

25 Zhang L, Xie M, Fu M. Assessment of age-related changes in left ventricular twist by two-dimensional ultrasound speckle tracking imaging. J Huazhong Univ Sci Technolog Med Sci 2007;27:691-5.

26 Zhang Y, Zhou QC, DR P. Differences in left ventricular twist related to age: speckle tracking echocardiographic data for healthy volunteers from neonate to age 70 years. Echocardiography 2010;27:1205-10.

27 Bertini M, Nucifora G, Marsan NA, et al. Left ventricular rotational mechanics in acute myocardial infarction and in chronic (ischemic and nonischemic) heart failure patients. Am J Cardiol 2009;103:1506-12. 